\title{
Direct Assembly of Large Area Nanoparticle Arrays
}

\author{
Heyou Zhang ${ }^{1}$, Calum Kinnear ${ }^{1}$, Jasper Cadusch², Timothy James ${ }^{2}$, Ann Roberts², \\ Paul Mulvaney ${ }^{1 *}$ \\ ${ }^{1}$ School of Chemistry \& Bio21 Institute, University of Melbourne, Parkville, Vic. 3010, Australia \\ ${ }^{2}$ School of Physics, University of Melbourne, Parkville, Vic. 3010, Australia \\ *e-mail:mulvaney@unimelb.edu.au
}

\begin{abstract}
A major goal of nanotechnology is the assembly of nanoscale building blocks into functional optical, electrical or chemical devices. Many of these applications depend on an ability to optically or electrically address a single nanoparticles. However, positioning large numbers of single nanocrystals with nanometre precision on a substrate for integration into solid-state devices, remains a fundamental roadblock. Here, we report fast, scalable assembly of thousands of single nanoparticles using electrophoretic deposition. We demonstrate that gold nanospheres, down to $30 \mathrm{~nm}$, and gold nanorods can be assembled into pre-defined patterns on transparent conductive substrates within a few seconds. We find that rod orientation can be preserved during deposition. We also show that arrays of nanorod dimers can be assembled with separations of down to $50 \mathrm{~nm}$. As proof of scale up, we demonstrate the scalability and fidelity of the process by creating centimetre scale patterns comprising more than 1 million gold nanoparticles.
\end{abstract}


Nanoscale particles exhibit unusual, size-dependent optical, luminescent, magnetic, catalytic or electronic properties. As a result, there is considerable scope for the development of diverse new applications for these materials including: nanoelectronics ${ }^{1}$, biosensors ${ }^{2}, 3$ ultrasensitive biodiagnostics $^{4}$, high-density information storage media ${ }^{5}$ and full-colour plasmonic pixels for displays $^{6}$. However, while much attention has focussed on the synthesis and characterisation of a wide range of nanocrystals over the last two decades, there has been less focus on the equally important challenge of nanocrystal assembly. The key challenge in nanoscale particle assembly is to precisely control assembly of individual molecular building blocks at the nanometre scale. These building blocks may be quantum dots, metal nanocrystals, biomolecules, single virus particles, polymers, DNA strands or hybrid inorganic-organic structures. The spacing between these nanoscale building elements is often quite critical. For example, by specifically controlling the distance between particles, plasmonic pixels made from aluminium nanorods are capable of producing a range of monochromatic colour responses ${ }^{6,7}$.

Thermal evaporation is one of the most commonly used techniques for creating arrays of nanostructures ${ }^{8}$. This method provides a "top-down" pathway for fabrication. However, thermal evaporation is restricted by the cost, the roughness of the deposited films and the limited array of suitable target materials 9 . In principle, directed, "bottom-up" assembly provides much greater flexibility $^{10}$. Directed assembly is a method to assemble existing nanoscale materials onto a prepatterned substrate by application of external forces. The goal is to maintain the original morphology of the materials during the fabrication and to use the external forces to push the materials into the pre-patterned templates. It is possible to apply this approach to differently shaped materials such as spheres, rods, stars and other complex particles such as alloys and core-shell materials. Moreover, by choosing suitable forces, directed assembly can be applied to a wide range of materials ${ }^{11,12,13}$. There are several promising sources of such external fields including capillary forces $^{14}$, chemical binding ${ }^{15}$ and electrophoretic forces ${ }^{16}$.

To date such assembly has only been demonstrated on small scales. For example, Kuemin et al. 17 and Shahrabi et al. ${ }^{18}$ successfully assembled gold nanorods (NRs) using capillary force assembly (CFA). By controlling the process parameters, particles were assembled and oriented into prepatterned substrates. However, CFA is extremely sensitive to surface roughness and to surface contaminants, which cause contact line pinning and to mis-assembly ${ }^{14}$. Another directed assembly method is chemically directed assembly (CDA). CDA exploits the attractive chemical interactions between particles and the substrate to effect assembly as demonstrated by Nepal et al ${ }^{15}$. Their approach relies on the particles sampling the well at some point along their trajectories and 
becoming adsorbed to the adhesive coating. In theory, this method is ideal for all kinds of material fabrication, as long as the particles and substrate have been functionalized properly. However, the deposition rates are often slow and non-specific adsorption leads to high levels of background noise. Creating chemistry that is specific for each type of particle-substrate combination is also challenging.

The third directed assembly approach is electrophoretic deposition (EPD). EPD has many advantages. For example it offers rapid processing and there is a little restriction on the particle type or type of substrate; it uses simple apparatus and is easy to apply ${ }^{19}$. A standard EPD process for $15 \mathrm{~nm}$ thin film fabrication only requires a few minutes. The thickness of the film can be precisely controlled by tuning the parameters of the EPD process such as applied voltage, particle concentration, and length of deposition time ${ }^{20}$. Furthermore, EPD has been reported as a versatile process for many kinds of materials, including ceramic powders ${ }^{21}$, metallic particles ${ }^{22}$, polymers ${ }^{23}$ and semiconductor materials ${ }^{24}$. Solomon and co-workers have studied the assembly of colloidal microcrystals ${ }^{25}$ and liquid crystals ${ }^{26}$ under direct current electric field. Prieve and co-workers have developed the fundamental theory for nanoparticle EPD assembly ${ }^{27} 28$. It thus appears that EPD could be a promising approach for nanomaterial fabrication. To date, most effort has focussed on large particle deposition. In 2009, Barbee et al. ${ }^{23}$ reported a method to deposit $500 \mathrm{~nm}$ polystyrene (PS) micro-beads into a $\mathrm{Ti}$ and $\mathrm{Au}$ coated silicon wafer with a patterned photoresist blocking material on the top. They successfully used EPD to directly deposit these micro-beads into an array. Later on the kinetics of this deposition process were also studied by Fang Qian et $\mathrm{al}^{29}$. They thoroughly investigated the key parameters during the PS deposition. They claimed that the voltage and particle-to-hole size ratio controlled the deposition quality of the PS beads. Those reports provided proof-of-concept that the combination of EPD and a patterned template is a promising technique for discrete particle assembly. However, extension to nanoscale assembly is highly challenging. Brownian motion disrupts particle deposition, while double layer forces dominate nanoparticle-surface dynamics. Furthermore, it is impossible in general, to watch single nanoparticles during deposition. 


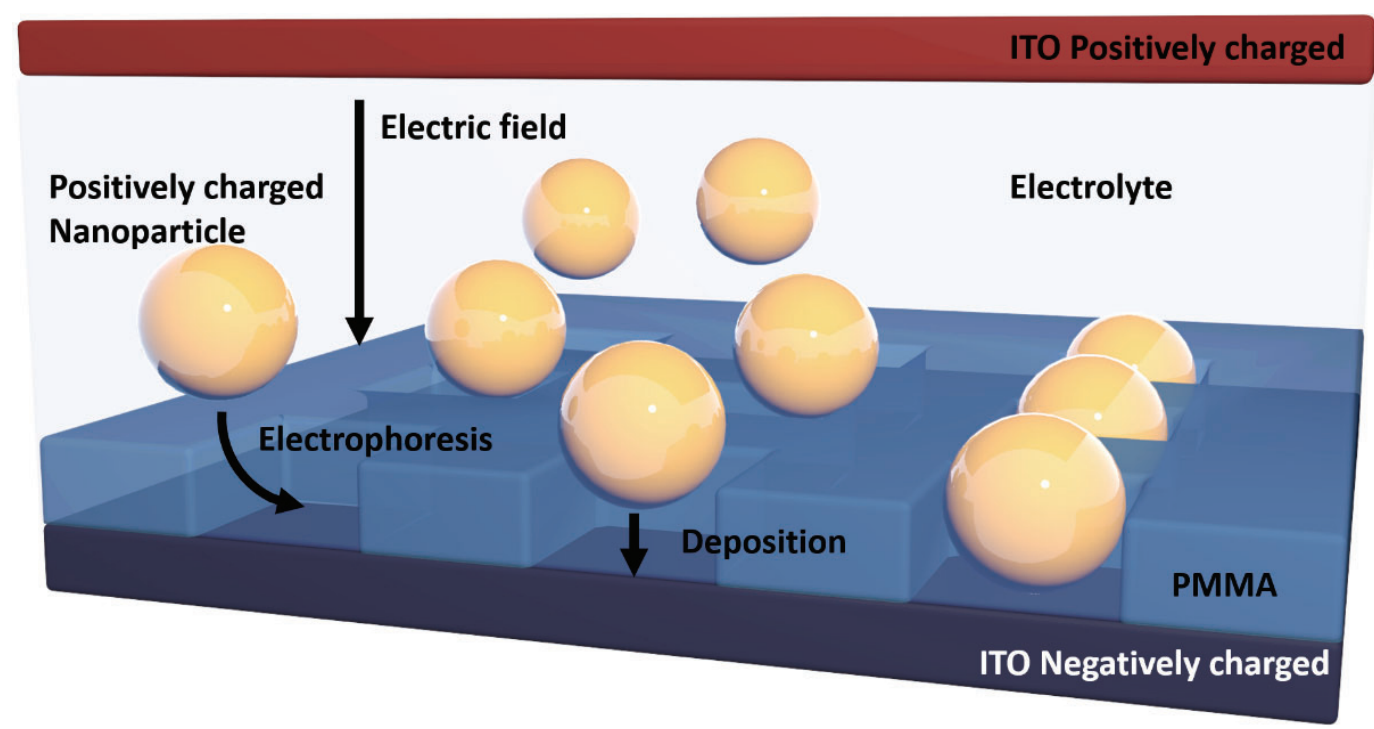

Figure 1. Schematic of the EPD process of nanospheres deposit onto EBL fabricated ITOPMMA template (dimensions not to scale). A controlled volume of nanoparticle colloidal solution mixed with controlled concentration of $\mathrm{NaCl}$ electrolyte is confined between an EBL patterned ITO-PMMA template and an ITO counter electrode on the top. Nanoparticles are positively charged by coating polydimethyl-diallyl ammonium chloride (poly-DADMAC). An electric field is generated by applying potential between the ITO-PMMA template and ITO counter electrode. Nanoparticles are influenced by electrophoresis and deposited into the cavities where there are not covered by PMMA on the template.

In this article, we demonstrate a highly efficient and ordered method to assemble single gold nanoparticles (spheres and rods) into a pre-patterned poly-methyl methacrylate (PMMA)-indium tin oxide (ITO) template via EPD. An EPD cell was designed to hold ITO and PMMA-ITO electrodes, and also containing positively charged gold nanoparticles and electrolyte colloid in between (Figure 1). A potential was applied on both ITO electrodes to generate the electric field. Under the influence of the electric field, particles are moving due to the electrophoresis and finally deposited on to the template. We designed the pattern structure by electron beam lithography (EBL) and studied the influence of key parameters on the EPD process. Remarkably, we find that EPD is self-terminating. Deposition of a nanoparticle into a surface well blocks the electric field and consequently only single particles are deposited.

The primary assembly of gold nanoparticle via EPD is carried by 40 x 40 square arrays with $120 \times 120 \mathrm{~nm}$ box cavities (Figure S2a). Figure 2a shows a dark field microscopy image of the array filled by $110 \mathrm{~nm}$ gold nanospheres (NSs). Since near-field optical coupling of nanoparticles is dependent on the gap between each particle and the separation of each cavity is $4 \mu \mathrm{m}$, we can clearly observe each individual particle inside the array. The array was nearly $100 \%$ filled by gold NSs with uniform yellowish scattering light, and non-specific binding was minimal. The scanning electron microscope image of part of a gold nanosphere array is shown in figure $2 \mathrm{~b}$. Each cavity 
was filled with only one particle. All nine gold NSs were located inside cavities. The single particle scattering spectrum was also measured for the deposited gold NSs. Figure S5a is the normalized scattering spectra for ten gold NSs in a single row. We randomly chose ten particles to verify the quality and consistency of EPD assembled gold NSs. The scattering spectra show a similar peak around $600 \mathrm{~nm}$, which is correlated to the reported scattering spectrum of $110 \mathrm{~nm}$ single gold $\mathrm{NS}^{30}$. A slight difference in the 10 spectra at $550 \mathrm{~nm}$ and $650 \mathrm{~nm}$ originates from the inhomogeneous distribution of particle sizes.
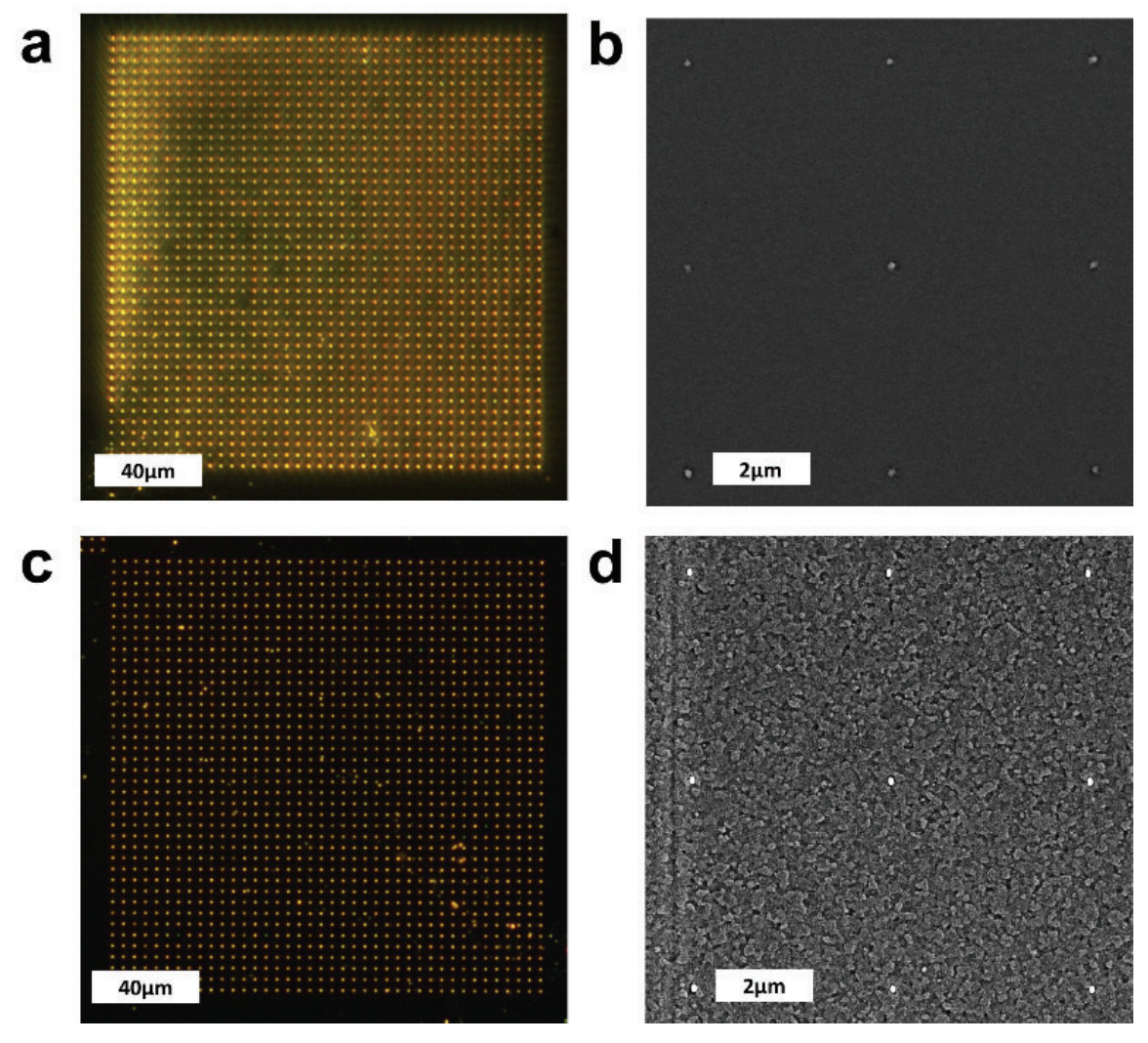

Figure 2. Results of gold NSs and gold NRs arrays fabricated via EPD direct assembly (a) Dark-field image of a $40 \times 40$ box array filled with 110nm gold NSs. (b) SEM image of several gold NSs in the array from Figure 1a. (c) Dark-field live image of a 40 x 40 gold NRs array filled by $100 \mathrm{~nm}$ x $40 \mathrm{~nm}$ gold NRs. (d). Scanning electron microscope image of several gold NRs in the array of Figure 1c.

Compared to gold NSs, gold NRs are more appealing in terms of the anisotropic optical properties and distinct colour effect. We also managed to assemble gold NRs with size of $100 \mathrm{~nm}$ in length and $40 \mathrm{~nm}$ in width via EPD. Figure $2 \mathrm{c}$ is a dark-field image of an array filled by $100 \times 40$ $\mathrm{nm}$ gold NRs. The design of the template is similar to the templates used for gold NSs deposition but with rod-like cavities (Figure S2b). The separation between each rod in the array is $4 \mu \mathrm{m}$, enabling individual rods to be easily distinguished by dark-field microscopy. The array was also nearly $100 \%$ filled by gold NRs with uniform scattering signals, due to the strong surface plasmon 
resonance at $720 \mathrm{~nm}$ for $100 \times 40 \mathrm{~nm}$ gold NRs under dark-field microscope, and only a tiny amount of background particles sticking as well. Figure $2 \mathrm{~d}$ is the scanning electron microscopy image of gold NRs arrays. It shows that gold NRs can be precisely deposited and aligned within cavities, and each cavity is filled by only one gold NR. All the gold NRs were aligned with the same orientation as the cavities. Clearly, the orientation of the cavities directs the orientation of the rods.
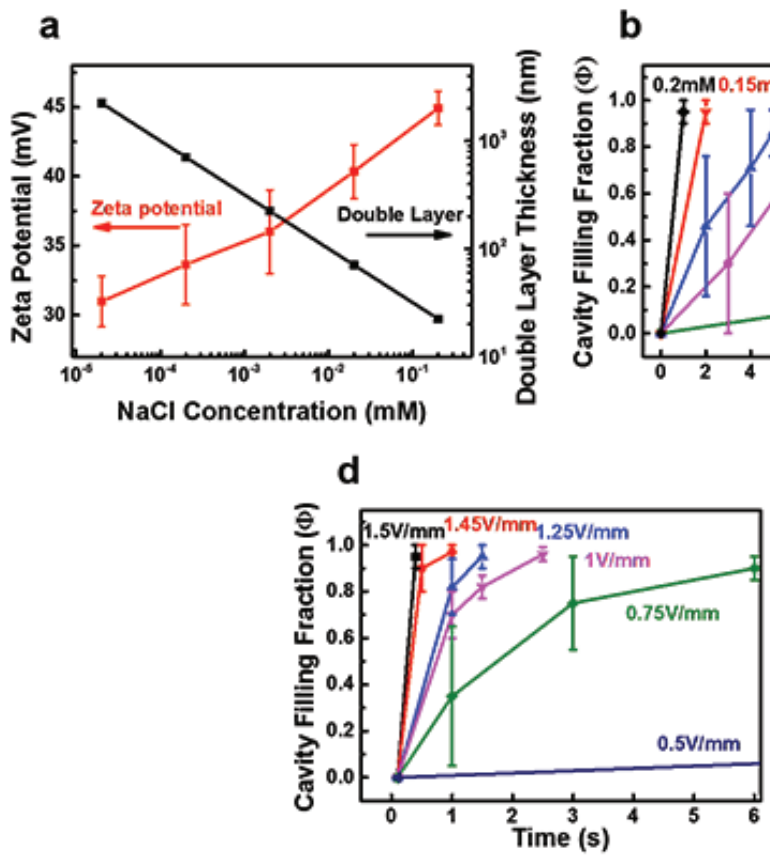

b
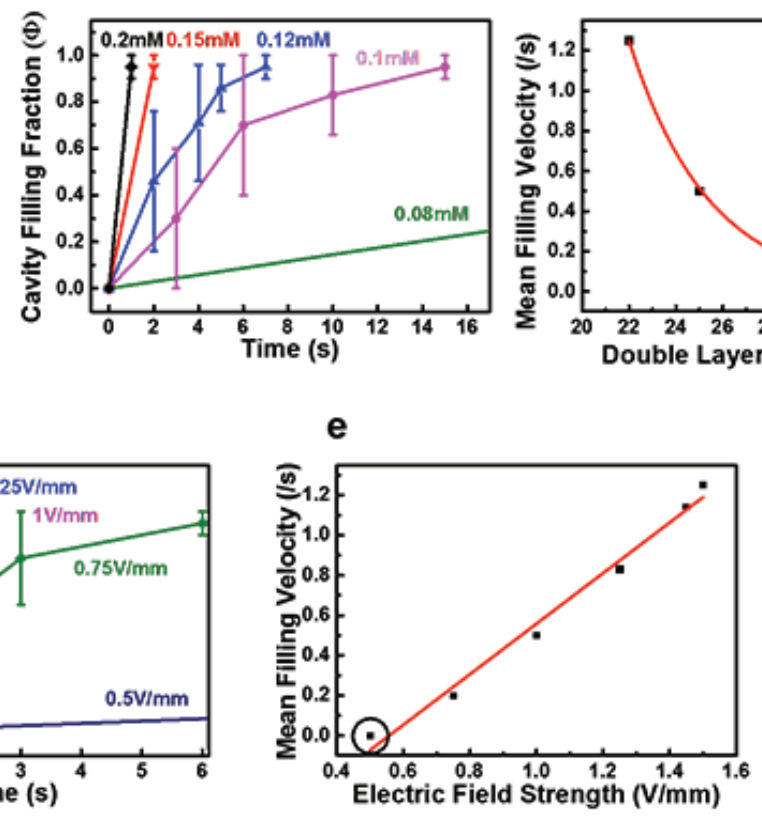

Figure 3. Parameter studies of EPD process. (a) The plot of zeta potential (left axis) and double layer thickness (right axis) as a function of $\mathrm{NaCl}$ concentration in $110 \mathrm{~nm}$ gold NSs solution. (room temperature 293K, $\mathrm{pH}=7$, p-DADMAC coated particles) (b) The plot of cavity filling fraction $\varphi$ as a function of time for different $\mathrm{NaCl}$ concentration (Electrode spacing $=2 \mathrm{~mm}$, applied potential $=3 \mathrm{~V}$, gold NSs concentration $=$ $30 \mathrm{ug} / \mathrm{mL}$, room temperature $293 \mathrm{~K}, \mathrm{pH}=7$, aqueous phase). (c) The plot of mean filling velocity as a function of double layer (d) the plot of cavity filling fraction $\varphi$ as a function of time for different electric field strength (Electrode spacing $=2 \mathrm{~mm}, \mathrm{NaCl}$ concentration $=0.2 \mathrm{mM}$, gold NSs concentration $=30 \mathrm{ug} / \mathrm{mL}$, room temperature $293 \mathrm{~K}, \mathrm{pH}=7$, aqueous phase). (e) The plot of mean filling velocity as a function of electric field strength. (All error bars indicate 5 standard deviations around the mean value for each data point).

To evaluate the consistency of this deposition, ten gold NRs from a row in the array were randomly chosen and their scattering spectra recorded (Figure S5b). The overlapping of all ten spectra indicates both the successful assembly of gold NRs and their high degree of monodispersity.

In order to understand and control the EPD assembly process, key parameters of gold nanoparticle EPD process are also investigated to optimize and predict the assembly. According to Smoluchowski ${ }^{31}$ equation (1),

$$
\mu=\frac{v}{E}=\frac{2}{3} \frac{\varepsilon_{0} \varepsilon_{r} \xi}{\eta} f(\kappa r)
$$


where the mobility of a particle $(\mu)$ is defined as its velocity $v$, divided by the strength of the applied electric field, E and $\varepsilon_{0}$ is the vacuum permittivity, $\varepsilon_{r}$ is relative permittivity of the solvent, $\eta$ is the viscosity of the solvent and $f(\kappa r)$ is a function, which depends on the particle radius $(r)$ and the electrical double layer thickness $\left(\kappa^{-1}\right)$. For aqueous electrophoresis, the mobility of a particle is influenced by (a) the applied electric field E, (b) the particle's zeta potential $\xi$ and (c) the double layer thickness of the particle $\left(\kappa^{-1}\right)$. Figure 3 a shows the changes in measured zeta potential and double layer thickness as a function of $\mathrm{NaCl}$ concentration. The double layer thickness is given by equation $(2)^{32}$ :

$$
\kappa^{-1}=\left(\frac{\varepsilon \varepsilon_{0} k T}{2 c z_{i}^{2} e_{0}^{2}}\right)^{\frac{1}{2}}
$$

Here $\mathrm{k}$ is the Boltzmann constant, $\mathrm{z}$ is the ion charge, $e_{0}$ is the elementary charge and $\mathrm{c}$ is the concentration of 1-1 electrolyte and $\kappa^{-1}$ is the double layer thickness. When the concentration of $\mathrm{NaCl}$ was increased, the thickness of the double layer decreased. Figure $3 \mathrm{~b}$ shows the influence of electrolyte concentration on the filling rate at a fixed gold particle concentration $(30 \mu \mathrm{g} / \mathrm{mL})$ and fixed electric field $(1.5 \mathrm{~V} / \mathrm{mm})$. When $0.2 \mathrm{mM}$ or $0.15 \mathrm{mM} \mathrm{NaCl}$ was present, the deposition occurred within 1 or 2 seconds (black and red lines in Figure 3b). However, when the $\mathrm{NaCl}$ concentration was reduced to $0.08 \mathrm{mM}$, the gold particles deposited much more slowly (blue, pink and green curves in Figure 3b). We further plotted the mean filling velocity and double layer thickness (Figure 3c) as a function of the double layer thickness. Increasing the electrolyte concentration in the EPD system increased the filling rate. However, within the range of $\mathrm{NaCl}$ concentration from $0.08 \mathrm{mM}$ to $0.2 \mathrm{mM}$, the zeta potential of gold colloid remained close to +44 $\mathrm{mV} \pm 2 \mathrm{mV}$. Hence, the main impact of changing the electrolyte concentration during EPD assembly is to decrease the double layer thickness, which facilitates egress of the gold particles into the nanocavities.

Another crucial factor for the EPD process is the applied potential. The time to completely fill all cavities decreased when the applied potential was increased (Figure 3d), as expected from equation (1). A linear relationship between the mean filling velocity and the electric field strength was observed, as shown in Figure 3e. Hence, the particle filling speed is proportional to the electric field as we expect from equation (1). However, when the applied potential was decreased to $0.5 \mathrm{~V} /$ mm (circled point in Figure 3e), there was no deposition. This means there is a "turn on" potential for EPD assembly. This "turn on" potential is necessary to overcome the Brownian motion of the nanoparticles. 
a

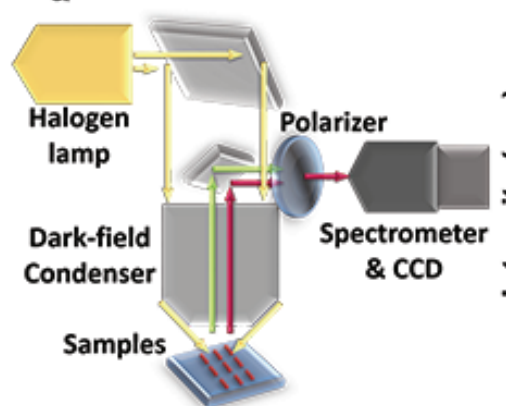

b

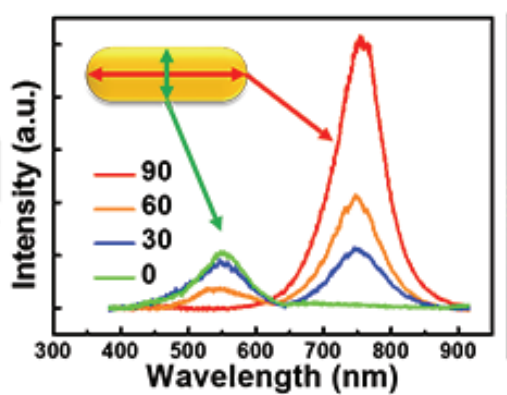

C

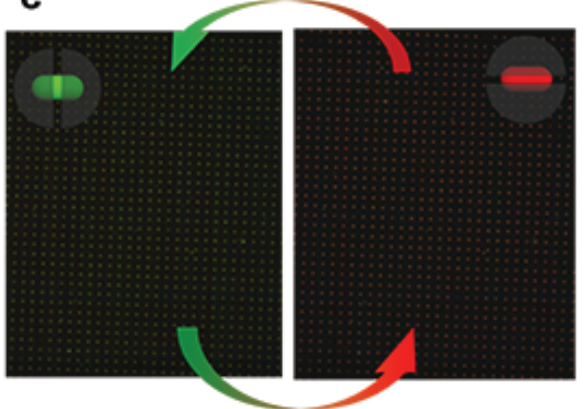

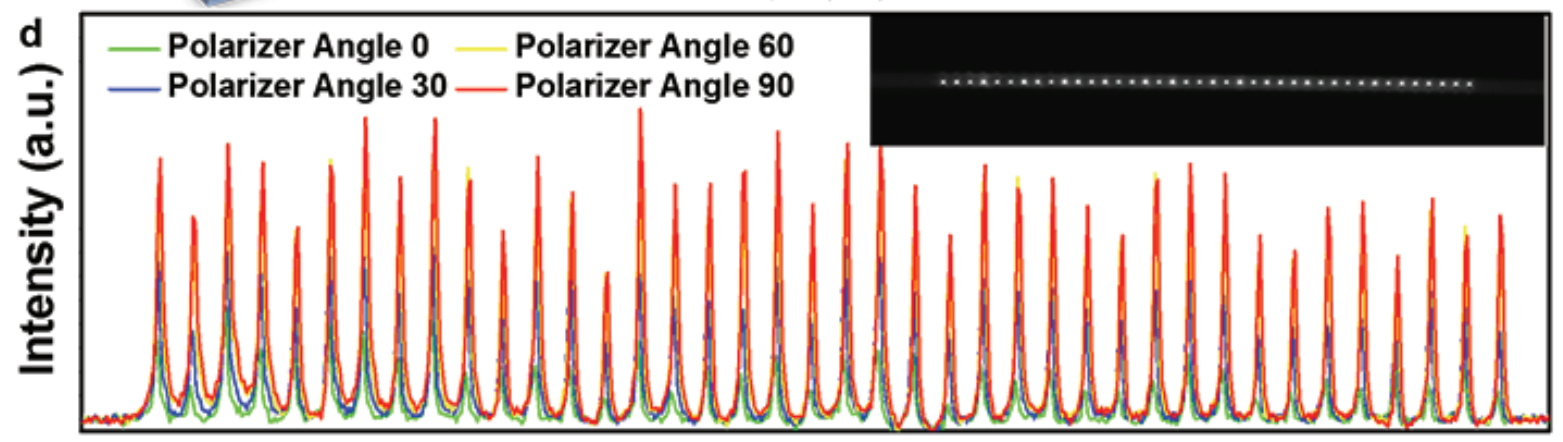

Particle Position

Figure 4. Polarization dependent optical properties of gold NRs array. (a) The schematic of reflective dark-field microscope attached with polarizer. (b)The scattering spectrum of single gold NRs under different polarization angles. (c) Dark-field images of gold NRs array under 0 and 90 degree polarization angles. (d) Overall intensity changes of a line of gold NRs via different polarization angles.

The quality of the gold NR assembly was investigated by polarized dark-field microscopy. Figure $4 \mathrm{a}$ is a schematic image of the set-up of the reflection dark-field microscope. Figure $4 \mathrm{~b}$ shows the intensity changes in the scattering from single gold NRs for a series of polarization angles. At 0 degrees, the polarizer is orthogonal to the major axis of the gold NR and therefore, the intensity of the transverse scattering peak dominates, causing the gold NRs to appear green (scattering peak at $530 \mathrm{~nm}$ ). Conversely, upon rotation of the polarizer to 90 degrees, the perceived colour of the gold NRs is dominated by the longitudinal surface plasmon oscillations and the rods appear red $(720 \mathrm{~nm})$. Because the cavities were all oriented in one direction, all the particles deposited in the cavities should follow the same polarization-dependent colour changes. This is demonstrated in Figure 4c. As we rotatd the polarizer from 0 to 90 degrees, the colour of the gold NRs uniformly changed from green (Figure 4c left) to red (Figure 4c right), respectively. This uniform colour change over the entire array indicates that the gold NRs were reproducibly deposited and aligned into cavities via EPD. To further prove the gold NR alignment, we used dark-field microscopy to investigate the intensity changes of a row of gold NRs in an array as shown in Figure 4d. When the polarizer was at 90 degrees, all 40 particles displayed the highest intensity (red curves). Upon rotation of the polarizer by 30 degrees to 60 degrees, the scattering intensity 
decreased correspondingly (yellow curves). A further shift to 30 degrees led to a further intensity decrease (Blue curves). Finally, when the polarizer was rotated to 0 degrees, the spectra exhibited the lowest intensity (Green curves). This polarization-dependent intensity change occurs because the longitudinal mode is far more intense than the transverse mode of the gold NRs. The uniform intensity change across a row of gold NRs indicates that they were all perfectly aligned with the same orientation.

a

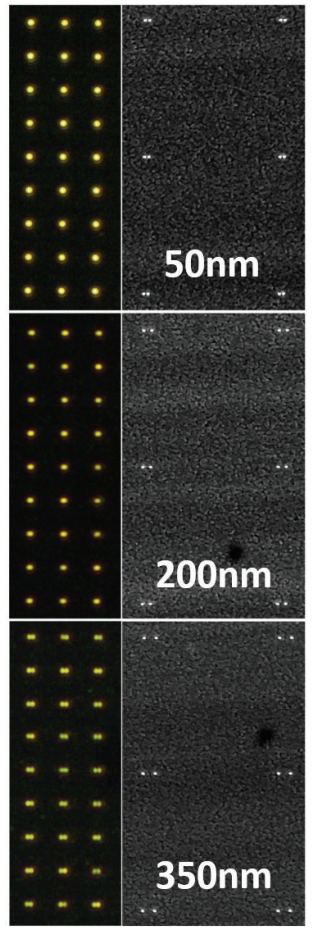

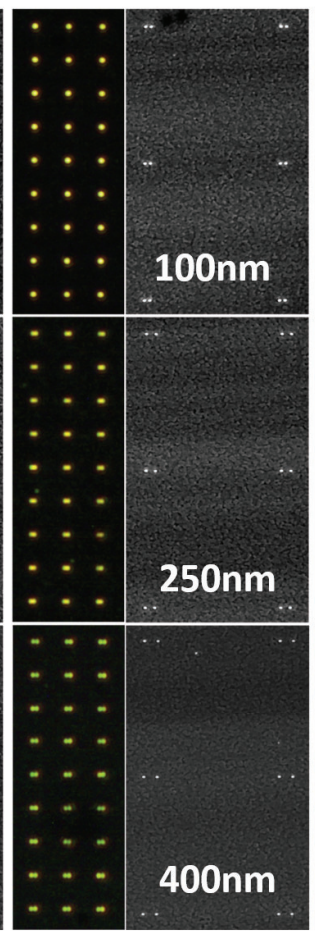
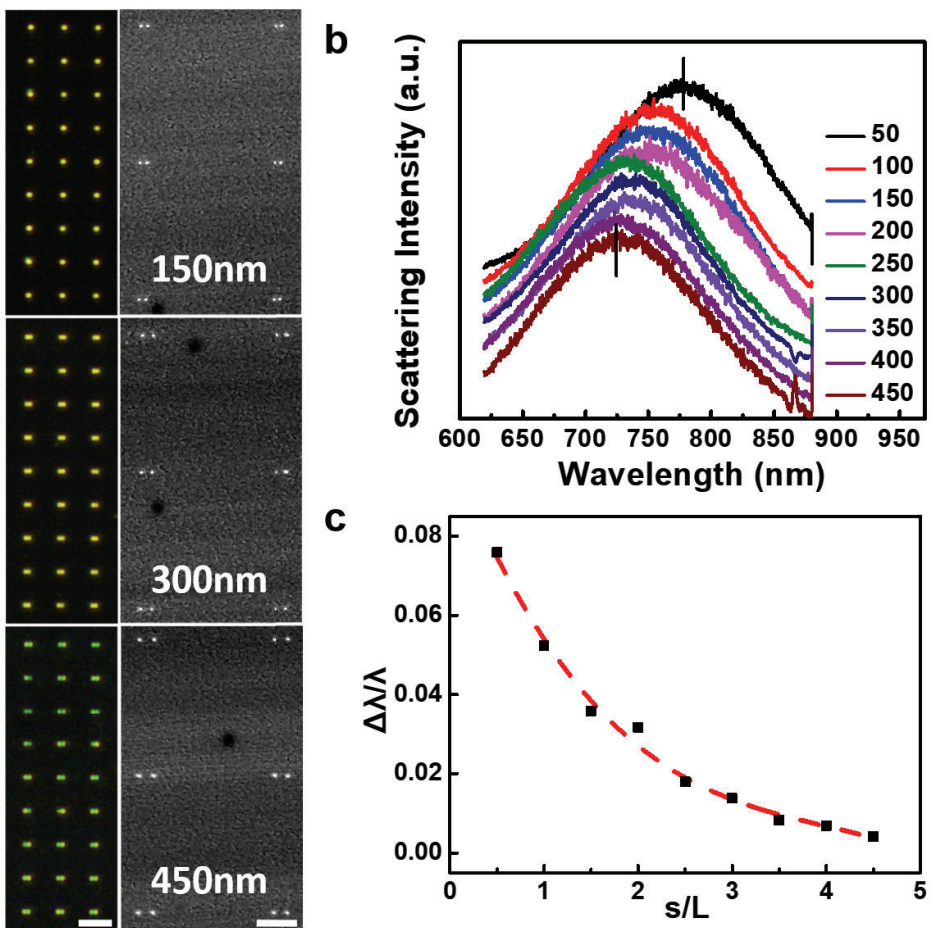

C

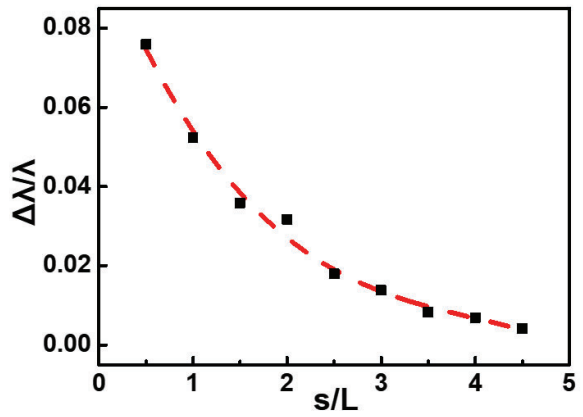

Figure 5. Dimer rod array assembled via EPD (a) Dark-field images (left) and corresponding scanning electron microscopy images (right) for tip to tip dimer gold NRs structure array deposited by EPD with the separation distance from $50 \mathrm{~nm}$ to $450 \mathrm{~nm}$. Scale bar $4 \mu \mathrm{m}$ (drak-field image) and $2 \mu \mathrm{m}$ (scanning electron microscopy). (b) Scattering spectra of dimer gold NRs structures with separation distance $50 \mathrm{~nm}$ (top) to $450 \mathrm{~nm}$ (bottom). Step: $50 \mathrm{~nm}$. (c) Plasmon band shift as a function of separation distance between particles determined experimentally (black square) and cubic fitting (red dash line).

To demonstrate that EPD can reproducibly generate large numbers of more complex structures, we assembled gold nanorod dimers with spacings between $50 \mathrm{~nm}$ and $400 \mathrm{~nm}$. Figure $5 \mathrm{a}$ presents a set of scanning electron microscopy images of dimer structures and associated dark-field images. Below the $200 \mathrm{~nm}$ separation distance, the two gold NRs were optically coupled, and appeared as a single spot under $50 \times$ magnification dark-field microscope. Above $250 \mathrm{~nm}$ separation distance, the dimer image started to split into two, and above $450 \mathrm{~nm}$ separation, the two particles appeared as distinct points of light. Figure $5 \mathrm{~b}$ shows the scattering spectra of dimers as a function of the 
separation distance. The series spectra exhibited a red shift from $\sim 730 \mathrm{~nm}$ to $\sim 780 \mathrm{~nm}$ as the separation distance was decreased. Furthermore, Figure 5c presents a plot of the SPR peak as a function of the normalised interparticle separation. The results were accurately fitted by a simple cubic function $^{33,34}$ :

$$
\Delta \lambda / \lambda \propto \frac{1}{(s / L)^{3}}
$$

where $\lambda$ is the plasmon peak wavelength of an isolated single gold nanorod, $\Delta \lambda$ is the shift in wavelength of a particular dimer structure compared to $\lambda, \mathrm{s}$ is the separation distance between gold NRs, and $\mathrm{L}$ is the length of the gold NRs. Above $\mathrm{s} / \mathrm{L}=4.5$, the plasmon wavelength is virtually identical to the single particle peak wavelength.
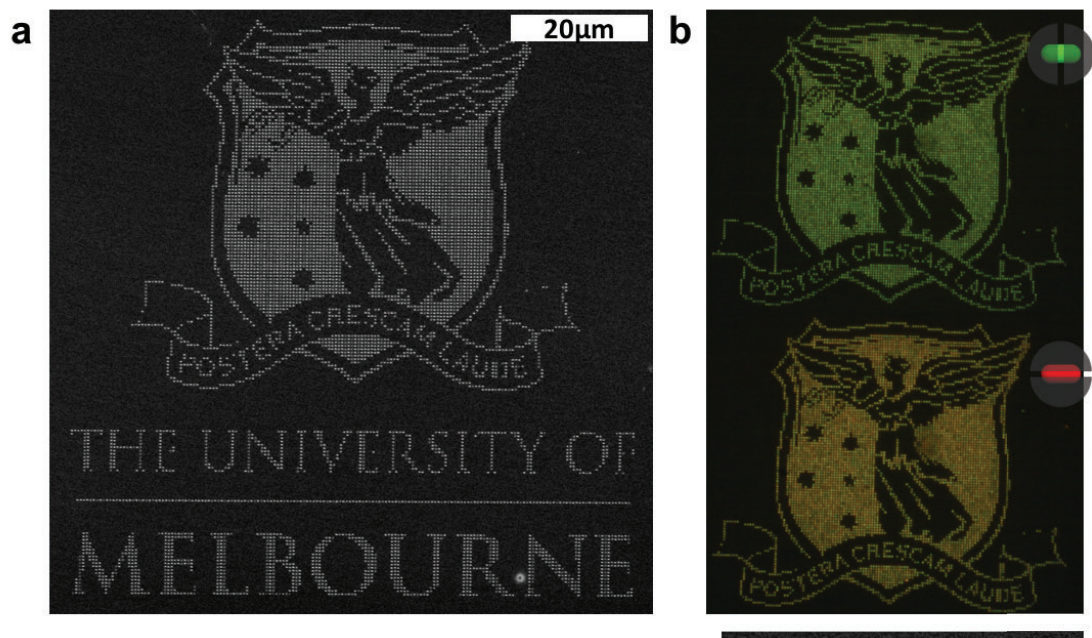

C
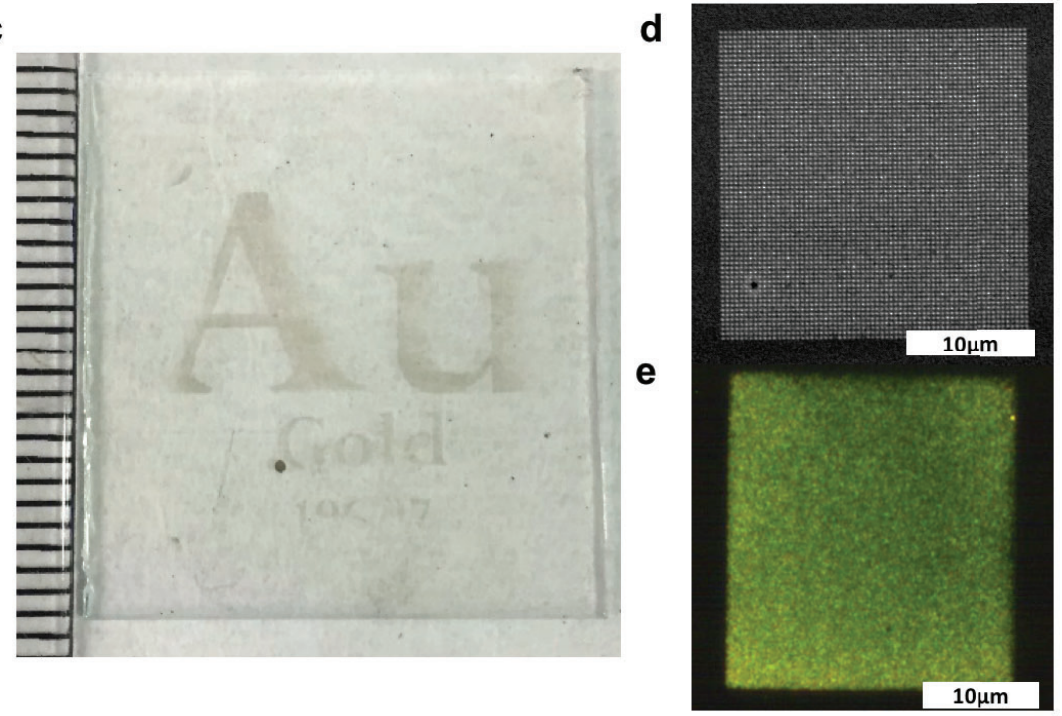

Figure 6. Large area Assembly (a) Scanning electron microscopy image of the logo of the University of Melbourne created from an array of single gold NRs. (b) Dark-field microscopy image of the University of Melbourne logo with vertical (top) and horizontal (bottom) polarization angles. (c) Digital picture of "Au" logo made by EPD on $25 \mathrm{~mm}$ x $25 \mathrm{~mm}$ ITO-PMMA glass slide. The left hand lines are $1 \mathrm{~mm}$ markings. (d) Scanning electron microscopy image of one 20 micron pixel of the "Au" logo showing a 66 x 66 array of single gold NRs. (e) Dark field microscopy image of one pixel of "Au" logo with 66 x 66 gold NRs filled. 
To demonstrate the fidelity and potential for scale-up, we have constructed an image of the University of Melbourne from single nanorods, shown in Figure 6a. The total scale of the logo is $100 \mu \mathrm{m}^{2}$ containing 6205 cavities. Figure S6 presents a set of high magnification scanning electron microscopy images of four parts of the logo filled by gold NRs. The cavities were filled by single gold NRs horizontally aligned. Again, a strong polarization dependence is observed under darkfield illumination (Figure 6b). The colour of the logo switches from red (longitudinal mode) to green (transverse mode) according to the angle of the polarizer. The ensemble scattering spectrum of the logo also shows a strong scattering intensity changes dependent on the polarizer angle (Figure S7).

Since the actual particle coverage in Figure 6a is much less than a monolayer and the entire logo is only $100 \mu \mathrm{m}$ across, the colour cannot be discerned by the naked eye. In order to fabricate a pattern which can be seen by the unaided eye, we designed and assembled an "Au" logo template with size $2.5 \mathrm{~cm} \times 2.5 \mathrm{~cm}$ (Figure 6c). This “Au" logo is composed of arrays of pixels. Each pixel is an array containing $66 \times 66$ cavities with a $300 \mathrm{~nm}$ separation distance between cavities and filled with gold nanorods. Figure 6d and Figure S8 are a set of scanning electron microscopy images of the "Au" logo at different magnifications. More than $98 \%$ of the array cavities are filled with single gold nanorods. Figure $6 \mathrm{~d}$ is a scanning electron microscope image showing one pixel of the gold NRs array. Because of the dense packing, the plasmon modes couple and the letters are visible in to the naked eye and in reflection evinces a dull gold colour. There are more than 1 million gold rods in this image, the first to be written in single nanoparticles. Figure 6e shows one pixel under the dark-field microscope. Due to the optical coupling of gold NRs in both longitudinal and transverse oscillation, the colour of one pixel appears greenish-yellow with a scattering peak around $580 \mathrm{~nm}^{33}$.

\section{Conclusions}

We have demonstrated that electrophoretic deposition can be used to assemble thousands, indeed millions, of single nanoparticles per second with nanometre spatial resolution into pre-patterned cavities or wells. The cavities can be used to control nanoparticle deposition by size, shape and orientation. The efficiency of the deposition process is very strongly dependent on the field strength and the electrolyte concentration. The accuracy of EPD assembly was also demonstrated by the reproducible fabrication of large numbers of gold rod dimers with spacings from $50 \mathrm{~nm}$ to $450 \mathrm{~nm}$. Scalability has also been demonstrated over 7 orders of magnitude with single particles being positioned within a few nanometres over centimetre areas. 


\section{Methods}

\section{Gold Nanocrystal synthesis}

Gold NSs with average $110 \mathrm{~nm}$ diameters were synthesized according to literature protocols ${ }^{30}$. Briefly, $20 \mathrm{~mL}$ ethylene glycol (Ajax Chemicals, 99.8\%) was added into a glass flask and stirred with a magnetic bar. Then $0.8 \mathrm{~mL}$ of $1 \mathrm{M} \mathrm{H}_{3} \mathrm{PO}_{4}$ solution and $0.4 \mathrm{~mL}$ of poly-dimethyl-diallyl ammonium chloride (poly-DADMAC) (Sigma-Aldrich, $20 \mathrm{wt} \%$ in $\mathrm{H}_{2} \mathrm{O}, \mathrm{Mw}=400,000-500,000$ ) were added into the flask. After 5 min stirring, $20 \mu \mathrm{L}$ of $0.5 \mathrm{M} \mathrm{HAuCl}_{4}$ aqueous solution (prepared by $\mathrm{HAuCl}_{4} \bullet 3 \mathrm{H}_{2} \mathrm{O}$ (Sigma-Aldrich)) was add and kept stirring for $15 \mathrm{~min}$ at room temperature. Then the solution was quickly loaded into a $195{ }^{\circ} \mathrm{C}$ oil bath for $30 \mathrm{~min}$ until the colour of solution changed from light yellow to colourless, purple and finally brown. After the reaction, the solution was loaded into a water bath and cooled down to room temperature. After the solution was thoroughly cooled down, $5 \mu \mathrm{L} 0.5 \mathrm{M} \mathrm{HAuCl}_{4}$ aqueous solution was added and kept stirring for 20 hours. The final solution was centrifuged at 9,000 rpm for $20 \mathrm{~min}$ and the precipitate was washed with ethanol and water three times at 3,000 rpm 5 min centrifugation. No further filtration was needed.

Gold NRs were synthesized following the binary surfactant method ${ }^{35}$. To create gold seeds, in a scintillation vial were added $5 \mathrm{~mL}$ of $0.5 \mathrm{mM} \mathrm{HAuCl}_{4}$ followed by $5 \mathrm{~mL}$ of $0.2 \mathrm{M}$ CTAB (SigmaAldrich, $\geq 98 \%$ ). Then, $0.3 \mathrm{~mL}$ of $20 \mathrm{mM}$ freshly prepared $\mathrm{NaBH}_{4}$ (Sigma-Aldrich, $\geq 98 \%$ ) was added under stirring (1,200 rpm). The solution was aged at room temperature for 2 hours before use. For gold NR growth, 9 g CTAB and 1.543 g NaOL (Sodium Oleate, Sigma-Aldrich, $\geq 99 \%$ ) were added into a $1 \mathrm{~L}$ flask with $250 \mathrm{~mL}$ water at $50{ }^{\circ} \mathrm{C}$ and mixed until dissolved. Then, the flask was set into a $30{ }^{\circ} \mathrm{C}$ water bath and $18 \mathrm{~mL}$ of $4 \mathrm{mM} \mathrm{AgNO}_{3}$ (Sigma-Aldrich, $\geq 99 \%$ ) were added. The solution was left undisturbed for $15 \mathrm{~min}$ at $30^{\circ} \mathrm{C}$ after which $250 \mathrm{~mL}$ of $1 \mathrm{mM} \mathrm{HAuCl}_{4}$ solution was added. The solution was kept stirring at $700 \mathrm{rpm}$ for 90 mins until colourless. $2.5 \mathrm{~mL} \mathrm{HCl}$ (32\%) was added to adjust the $\mathrm{pH}$. After $15 \mathrm{~min}$ of $400 \mathrm{rpm}$ stirring, $1.25 \mathrm{~mL}$ freshly prepared 0.1 M L-ascorbic acid (Sigma-Aldrich, reagent grade) were added to the solution, followed by vigorous stirring for $30 \mathrm{~s}$. Finally, $0.4 \mathrm{~mL}$ gold seed solution were added and the solution was then left undisturbed overnight at $30{ }^{\circ} \mathrm{C}$. To coat the gold NRs with p-DADMAC, the gold NRs solution were washed in water (under $4000 \mathrm{rpm}$ centrifugation). A 1\% p-DADMAC solution was added to the precipitate and sonicated for 10 mins. Finally, the solution was washed in water (at $9000 \mathrm{rpm}$ ) another 3 times. The structural characterization of gold NSs and gold NRs is shown in Figure S1. 


\section{Template Patterning}

The templates were patterned via electron-beam lithography (EBL). To prepare the mother template, polished ITO-glass slides (Delta Technologies, CG-51IN-S115) were used as the substrate. The ITO slides were thoroughly cleaned by consecutive sonication in ethanol, IPA (isopropanol) and water. Then a $50 \mathrm{~nm}$ PMMA blocking polymer layer was prepared by spin coating PMMA (MicroChem) at 1,000 rpm onto the ITO and baked at $180{ }^{\circ} \mathrm{C}$ for 5 mins. A thin layer of chromium was then evaporated onto the ITO slide using a thermal evaporator (Intlvac Nanochrome II) and stored in a dust-free environment for further use. To pattern the ITO-PMMA template, the slides were exposed to an electron-beam (Vistec EBPG 5000plaus ES) with a custom pattern created using modelling software (Klayout). After that, the chromium layer was removed in $5 \% \mathrm{H}_{2} \mathrm{SO}_{4}$ followed by development in solution with mixed methyl isobutyl ketone (MIBK) and isopropanol (IPA) $(\mathrm{MIBK}: \mathrm{IPA}=3: 1)$ for $1 \mathrm{~min}$ and the slide was finally rinsed with IPA three more times. The array template was characterized by Atomic Force Microscopy (MFP 3D, Asylum research) as shown in Figure S2. The thickness of the PMMA was always maintained 50-60 nm.

\section{EPD Cell assembly}

The EPD process was carried in a 3D printed cell (UP-Plus). It was made from acrylonitrile butadiene styrene polymer (ABS). The printing layer thickness was $0.2 \mathrm{~mm}$ with a 45 -degree printing angle. This cell was designed to fit into the dark-field microscope. There were two optical windows on the top and bottom panels, which enabled real-time observation of EPD under a darkfield microscope during the EPD process. A fixed chamber with a size of $8 \mathrm{~mm}(\mathrm{~L}) \times 8 \mathrm{~mm}(\mathrm{~W}) \times 2$ $\mathrm{mm}(\mathrm{H})$ was placed in the middle part. Two flow channels were connected to the chamber for gold colloid injection. The assembly of the EPD cell is shown in Figure S3. The patterned template and counter electrode were connected by copper tape with two copper wires separately. To avoid fluid leakage, the cell was sealed by putting Teflon tape between the cell and slides. The cell was finally tightened with four screws at the corners.

For large area EPD assembly, most of the cell assembly processes are the same as those used in the small-area EPD cell, except that multiple electrical contacts were required to minimize the IR drop across the ITO electrode. The EPD chamber was changed to $20 \mathrm{~mm}(\mathrm{~L}) \times 20 \mathrm{~mm}(\mathrm{~W}) \times 2 \mathrm{~mm}(\mathrm{H})$. 8 wires were hooked around both electrodes to generate an even electrode field across the template (Figure S4). For the EPD process, both electrodes were directly connected to a potentiostat (Keithley 2400 Source Meter). The patterned template was connected to the cathode and the counter electrode was connected to the anode. An approximately $0.3 \mathrm{~mL}$ gold colloid solution was injected 
into the flow-through channel. To initiate the EPD process, a direct current with constant potential was applied through the cell. Finally, to remove excess gold colloid, the template was rinsed by IPA and water, then dried by nitrogen blowing.

\section{Dark-field scattering spectroscopy}

The scattering spectra of single gold nanocrystals were collected with a Nikon Lv100 Eclipse inverted microscope, equipped with a dark-field condenser and LU plus ELWD 50×/0.55 Nikon Lens in the reflection configuration. The light source of the microscope was a $100 \mathrm{~W}$ quartz halogen lamp. The scattered light was captured by an Acton Micro-Spec 2150i imaging microscopy spectrograph fitted with a PIXIS 1024F CCD. Another CCD Camera (ThorLabs) was attached to the microscope to capture the dark-field images. A polarizer was placed in the pathway of the reflected light for polarization-dependence measurements.

\section{Electron microscopy and Atomic force microscopy}

All electron microscopy images were collected at the Bio21 Advanced Microscopy Facility (the University of Melbourne). For transmission electron microscopy (TEM) images, gold nanoparticle colloids were prepared on carbon coating, 300 mash copper grids (Electron Microscopy Sciences). Samples were imaged on the Tecnai F20 (FEI) under 200kV high voltage. Scanning electron microscopy (SEM) images were collected on an FEI Nova Nanolab (FEI) at $5 \mathrm{kV}$ high voltage and $1.6 \mathrm{nA}$ current. The atomic force microscopy (AFM) images were collected with an MFP-3D AFM (Asylum Research) loaded with silicon cantilevers (AC240TS-R3, Asylum Research).

\section{Acknowledgements}

The authors thank the ARC for support through grants LF110100161 and CE170100026. We thank the Melbourne Centre for Nanofabrication for access to EBL.

\section{Author contributions}

HZ designed the experiments and experimental devices, built the experiment set-up, performed the assembly experiments, and co-wrote the manuscript. CK synthesised gold nanoparticles, designed the surface chemistry and assisted with assembly experiments, and helped write manuscript. JC and TJ carried out nanofabrication of the ITO-PMMA template by EBL. AR oversaw the optics and helped write the manuscript. PM conceived the research, supervised the research work and co-wrote the manuscript. 
Additional information

Supplementary information is available.

\section{Competing financial interests}

The authors declare no competing financial interests. 


\section{References}

1. Rivest JB, Jain PK. Cation exchange on the nanoscale: an emerging technique for new material synthesis, device fabrication, and chemical sensing. Chemical Society Reviews 2013, 42(1): 89-96.

2. Liu J, Lu Y. A colorimetric lead biosensor using DNAzyme-directed assembly of gold nanoparticles. Journal of the American Chemical Society 2003, 125(22): 6642-6643.

3. Pingarrón JM, Yañez-Sedeño P, González-Cortés A. Gold nanoparticle-based electrochemical biosensors. Electrochimica Acta 2008, 53(19): 5848-5866.

4. Collins SS, Cittadini M, Pecharromán C, Martucci A, Mulvaney P. Hydrogen Spillover between Single Gold Nanorods and Metal Oxide Supports: A Surface Plasmon Spectroscopy Study. ACS nano 2015, 9(8): 7846-7856.

5. Zijlstra P, Chon JW, Gu M. Five-dimensional optical recording mediated by surface plasmons in gold nanorods. Nature 2009, 459(7245): 410.

6. James TD, Mulvaney P, Roberts A. The Plasmonic Pixel: large area, wide gamut color reproduction using aluminum nanostructures. Nano letters 2016.

7. Olson J, Manjavacas A, Basu T, Huang D, Schlather AE, Zheng B, et al. High Chromaticity Aluminum Plasmonic Pixels for Active Liquid Crystal Displays. ACS nano 2015, 10(1): 1108-1117.

8. Dai ZR, Pan ZW, Wang ZL. Novel nanostructures of functional oxides synthesized by thermal evaporation. Advanced Functional Materials 2003, 13(1): 9-24.

9. Brinker CJ, Lu Y, Sellinger A, Fan H. Evaporation-induced self-assembly: nanostructures made easy. Advanced materials 1999, 11(7): 579-585.

10. Li M, Bhiladvala RB, Morrow TJ, Sioss JA, Lew K-K, Redwing JM, et al. Bottom-up assembly of large-area nanowire resonator arrays. Nature Nanotechnology 2008, 3(2): 88-92.

11. Xiao J, Li Z, Ye X, Ma Y, Qi L. Self-assembly of gold nanorods into vertically aligned, rectangular microplates with a supercrystalline structure. Nanoscale 2014, 6(2): 996-1004.

12. Kosiorek A, Kandulski W, Glaczynska H, Giersig M. Fabrication of nanoscale rings, dots, and rods by combining shadow nanosphere lithography and annealed polystyrene nanosphere masks. Small 2005, 1(4): 439-444.

13. Pardo DA, Jabbour GE, Peyghambarian N. Application of Screen Printing in the Fabrication of Organic Light-Emitting Devices. Advanced Materials 2000, 12(17): 1249-1252.

14. Kuemin C, Stutz R, Spencer ND, Wolf H. Precise placement of gold nanorods by capillary assembly. Langmuir 2011, 27(10): 6305-6310.

15. Nepal D, Onses MS, Park K, Jespersen M, Thode CJ, Nealey PF, et al. Control over position, orientation, and spacing of arrays of gold nanorods using chemically nanopatterned surfaces and tailored particle-particle-surface interactions. ACS nano 2012, 6(6): 5693-5701.

16. Bavykin DV, Passoni L, Walsh FC. Hierarchical tube-in-tube structures prepared by electrophoretic deposition of nanostructured titanates into a TiO 2 nanotube array. Chemical Communications 2013, 49(62): 7007-7009.

17. Kuemin C, Nowack L, Bozano L, Spencer ND, Wolf H. Oriented Assembly of Gold Nanorods on the Single-Particle Level. Advanced Functional Materials 2012, 22(4): 702-708.

18. Flauraud V, Mastrangeli M, Bernasconi GD, Butet J, Alexander DT, Shahrabi E, et al. Nanoscale topographical control of capillary assembly of nanoparticles. Nature Nanotechnology 2017, 12(1): 73-80.

19. Sarkar P, Nicholson PS. Electrophoretic deposition (EPD): mechanisms, kinetics, and application to ceramics. Journal of the American Ceramic Society 1996, 79(8): 1987-2002.

20. Song KW, Costi R, Bulović V. Electrophoretic Deposition of CdSe/ZnS Quantum Dots for Light-Emitting Devices. Advanced Materials 2013, 25(10): 1420-1423.

21. Sarkar P, Haung X, Nicholson PS. Structural ceramic microlaminates by electrophoretic deposition. Journal of the American Ceramic Society 1992, 75(10): 2907-2909.

22. Giersig M, Mulvaney P. Preparation of ordered colloid monolayers by electrophoretic 
deposition. Langmuir 1993, 9(12): 3408-3413.

23. Barbee KD, Hsiao AP, Heller MJ, Huang X. Electric field directed assembly of high-density microbead arrays. Lab on a Chip 2009, 9(22): 3268-3274.

24. Brown P, Kamat PV. Quantum Dot Solar Cells. Electrophoretic Deposition of CdSe- C60 Composite Films and Capture of Photogenerated Electrons with n C60 Cluster Shell. Journal of the American Chemical Society 2008, 130(28): 8890-8891.

25. Shah AA, Ganesan M, Jocz J, Solomon MJ. Direct Current Electric Field Assembly of Colloidal Crystals Displaying Reversible Structural Color. ACS nano 2014, 8(8): 8095-8103.

26. Shah AA, Kang H, Kohlstedt KL, Ahn KH, Glotzer SC, Monroe CW, et al. Liquid crystal order in colloidal suspensions of spheroidal particles by direct current electric field assembly. Small 2012, 8(10): 1551-1562.

27. Prieve D, Anderson J, Ebel J, Lowell M. Motion of a particle generated by chemical gradients. Part 2. Electrolytes. Journal of Fluid Mechanics 1984, 148: 247-269.

28. Prieve DC, Sides PJ, Wirth CL. 2-D assembly of colloidal particles on a planar electrode. Current Opinion in Colloid \& Interface Science 2010, 15(3): 160-174.

29. Qian F, Pascall AJ, Bora M, Han TY-J, Guo S, Ly SS, et al. On-Demand and Location Selective Particle Assembly via Electrophoretic Deposition for Fabricating Structures with Particleto-Particle Precision. Langmuir 2015, 31(12): 3563-3568.

30. Lee Y-J, Schade NB, Sun L, Fan JA, Bae DR, Mariscal MM, et al. Ultrasmooth, highly spherical monocrystalline gold particles for precision plasmonics. ACS nano 2013, 7(12): 1106411070.

31. van Smoluchowski M. Versuch einer mathematischen theorie der koagulation kinetic kolloider losungen. Zeit Phys Chem 1917, 92: 129-168.

32. Besra L, Liu M. A review on fundamentals and applications of electrophoretic deposition (EPD). Progress in materials science 2007, 52(1): 1-61.

33. Funston AM, Novo C, Davis TJ, Mulvaney P. Plasmon coupling of gold nanorods at short distances and in different geometries. Nano letters 2009, 9(4): 1651-1658.

34. Tabor C, Van Haute D, El-Sayed MA. Effect of orientation on plasmonic coupling between gold nanorods. Acs Nano 2009, 3(11): 3670-3678.

35. Ye X, Zheng $\mathrm{C}$, Chen J, Gao Y, Murray CB. Using binary surfactant mixtures to simultaneously improve the dimensional tunability and monodispersity in the seeded growth of gold nanorods. Nano letters 2013, 13(2): 765-771. 


\section{Supporting Information}

\section{Direct Assembly of Large Area Nanoparticle Arrays}

Heyou Zhang ${ }^{1}$, Calum Kinnear ${ }^{1}$, Jasper Cadusch ${ }^{2}$, Timothy James ${ }^{2}$, Ann Roberts ${ }^{2}$, Paul Mulvaney ${ }^{1 *}$

${ }^{1}$ School of Chemistry \& Bio21 Institute, University of Melbourne, Parkville, Vic. 3010, Australia

${ }^{2}$ School of Physics, University of Melbourne, Parkville, Vic. 3010, Australia

*e-mail: mulvaney@unimelb.edu.au 


\section{Structural Characterization of Gold NSs and Gold NRs}
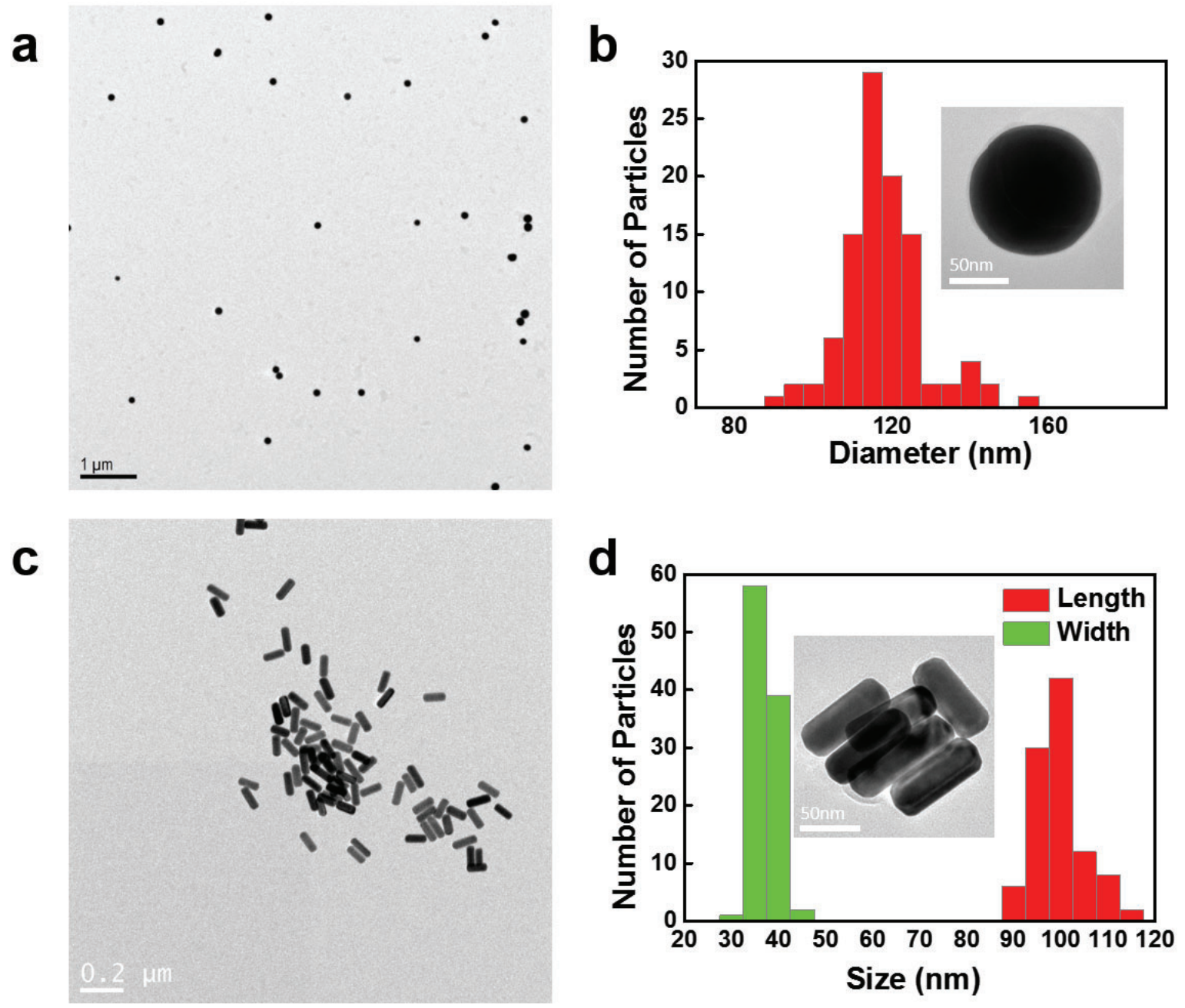

Figure S1. The transmission electron microscope images for (a) gold NSs with (b) $110 \mathrm{~nm}$ diameter distribution and (c) gold NRs with (d) $100 \mathrm{~nm}$ in length and 40nm in width distribution. 
The atomic force microscopy images for box and rod templates.
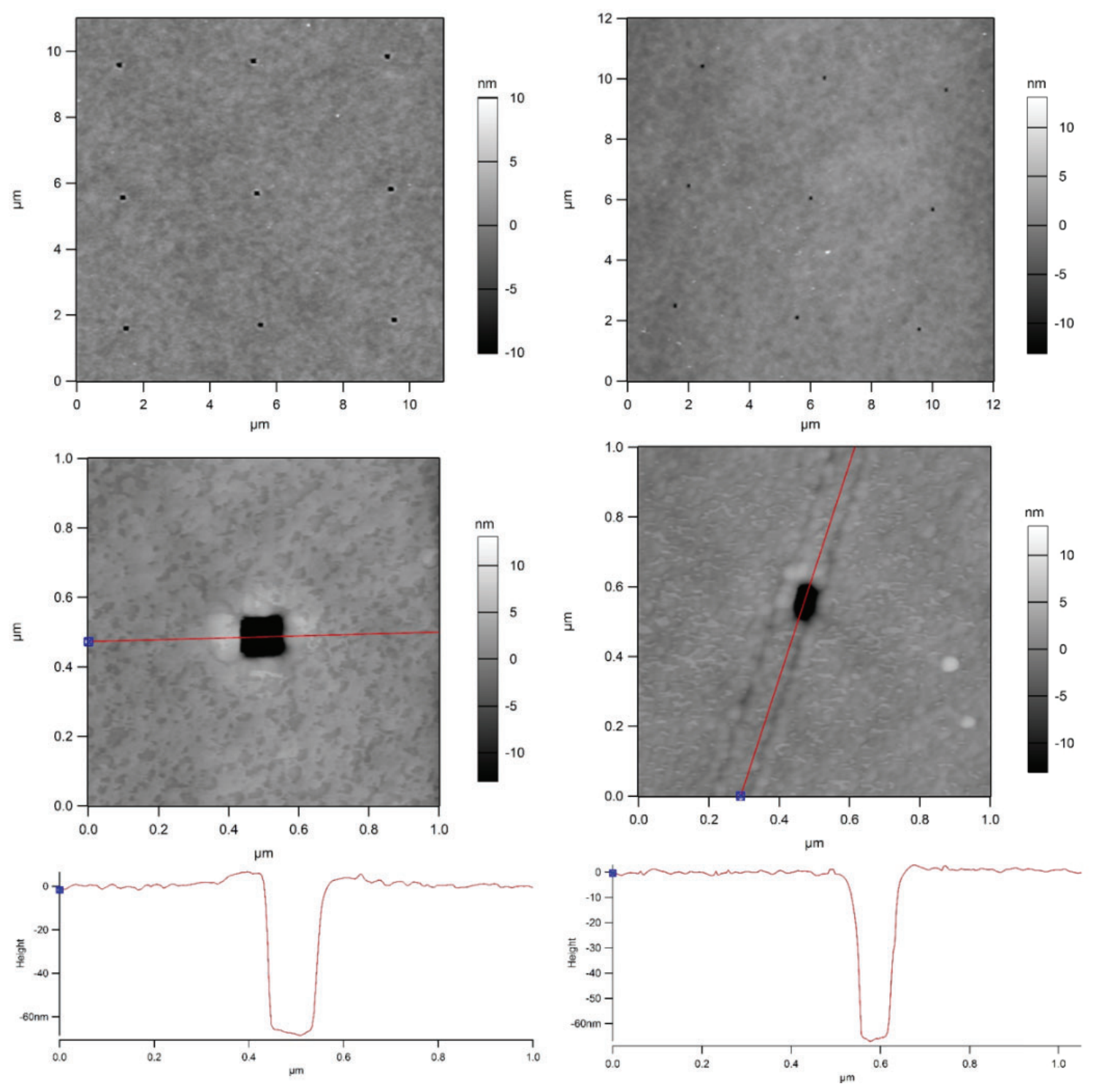

Figure S2. The atomic force microscopy images for box array (Left) and rod array (Right) and the height profile which shows 60nm PMMA thickness. 


\section{$\underline{3 \mathrm{D} \text { printed cells for EPD and its assembly }}$}
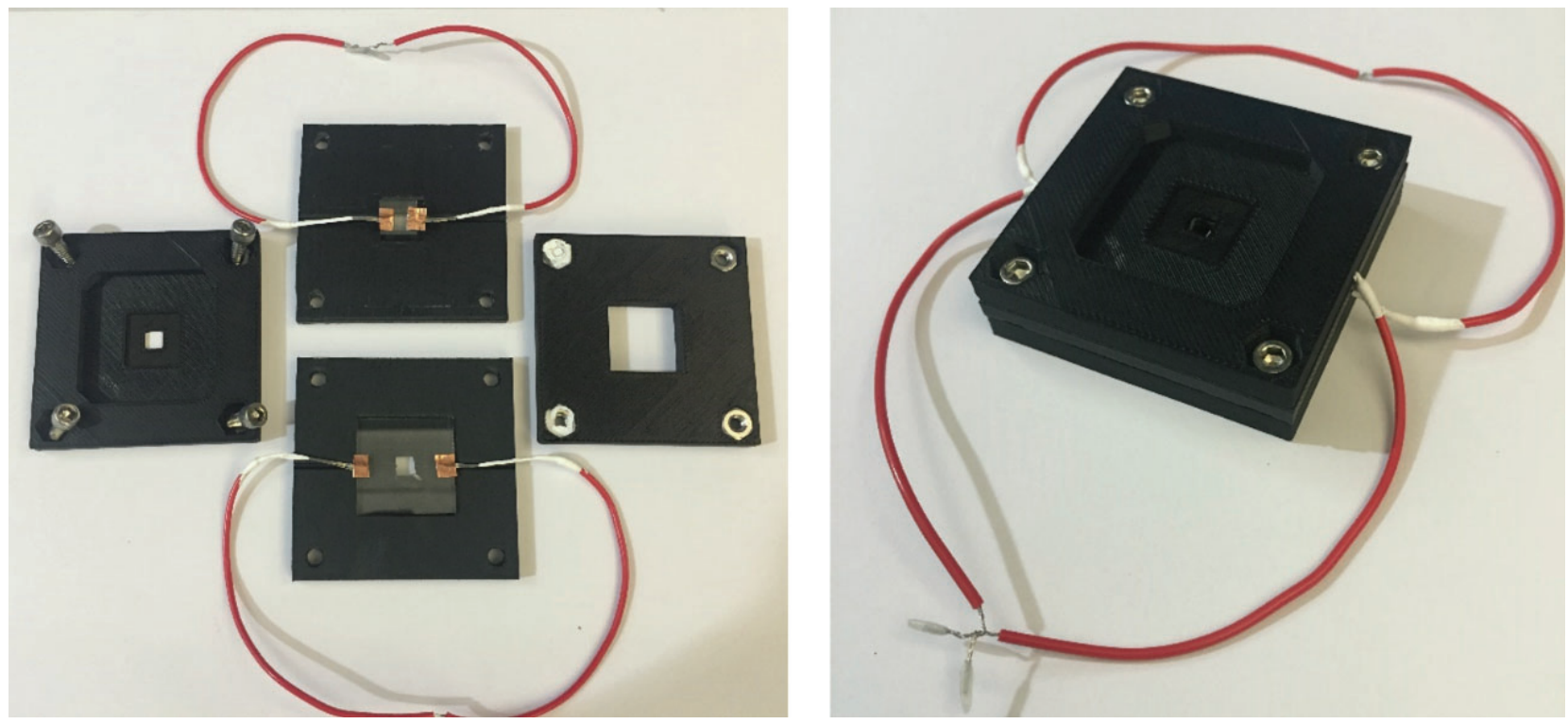

Figure S3. Digital images of assembly method and 3D printed, small-scale EPD cell with $10 \mathrm{~mm}(\mathrm{~L}) \mathrm{x}$ $10 \mathrm{~mm}(\mathrm{~W}) \times 2 \mathrm{~mm}(\mathrm{H})$ tank. Before and after assembly.
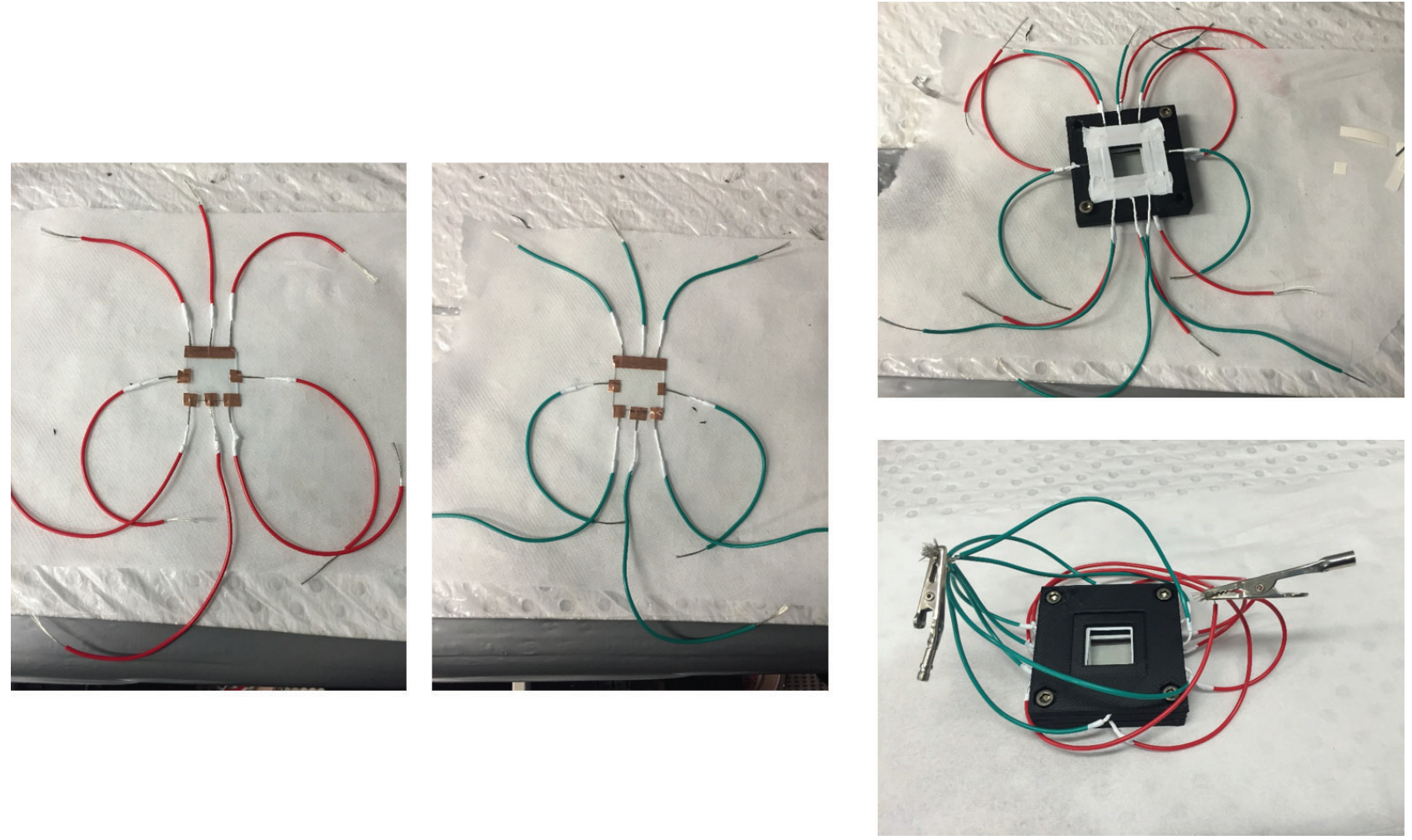

Figure S4. Digital images of assemble method and 3D printed large scale EPD cell with $20 \mathrm{~mm}$ (L) $\mathrm{x}$ $20 \mathrm{~mm}(\mathrm{~W}) \times 2 \mathrm{~mm}(\mathrm{H})$ tank. 


\section{Scattering spectrum of single gold nanoparticles in arrays}

a

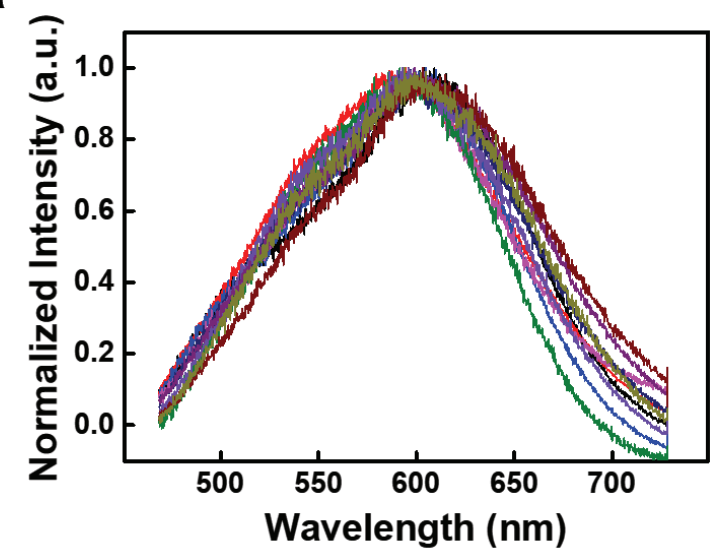

b

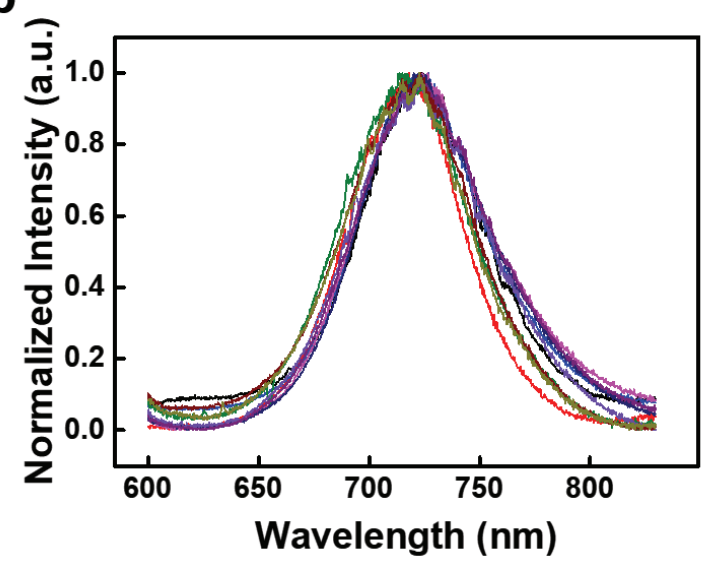

Figure S5. Single particle scattering spectra of gold NSs and gold NRs assembled in arrays via EPD. (a) Scattering spectrum shows the consistency for randomly ten gold NSs in a line of array. (b) Scattering spectrum shows the consistency for randomly ten gold NRs in a line of array 


\section{The logo of the University of Melbourne filled by gold NRs via EPD assembly.}

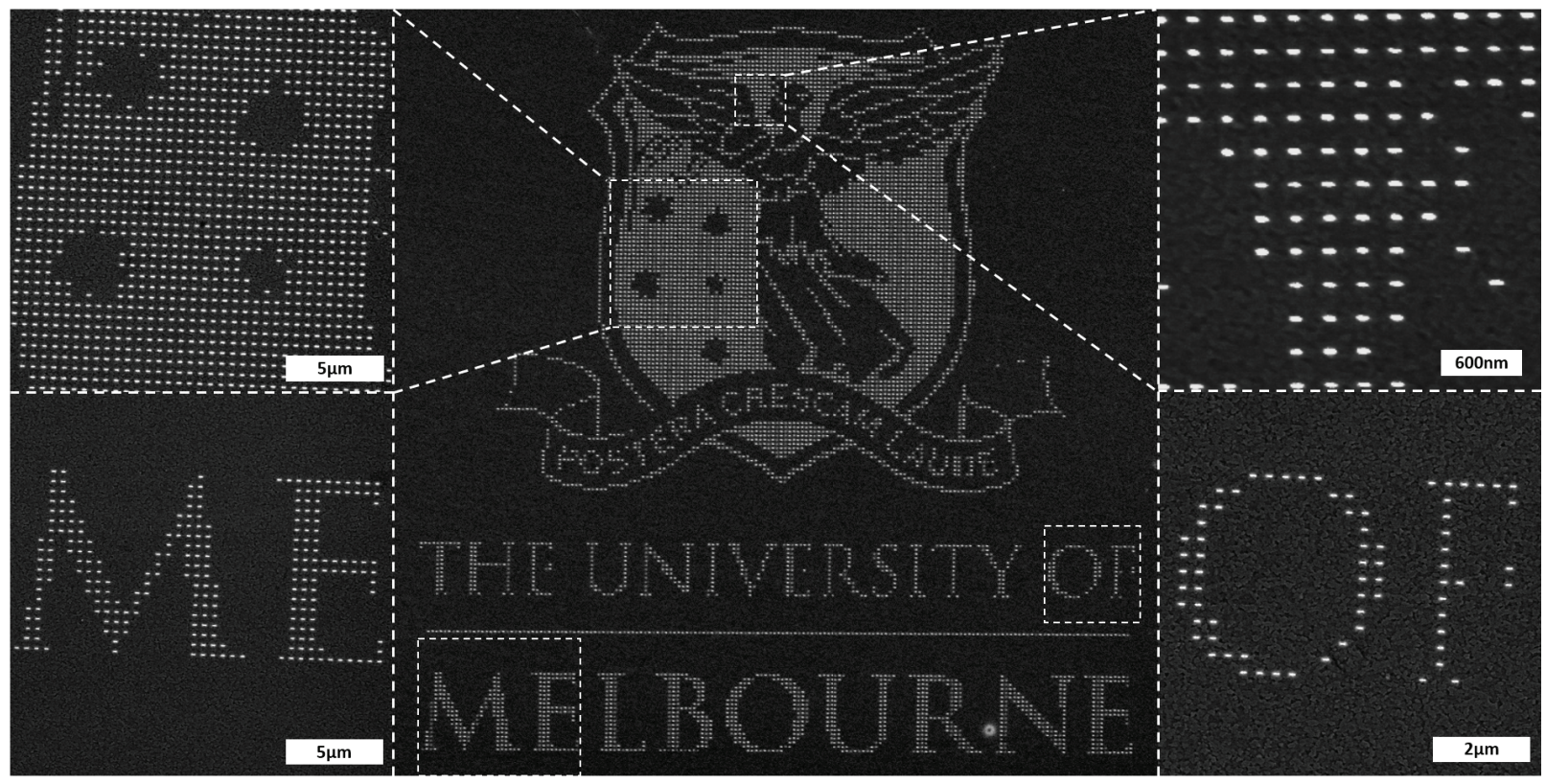

Figure S6. Scanning electron microscopy images of the logo of the University of Melbourne and high magnification scanning electron microscopy images of four parts in the logo.

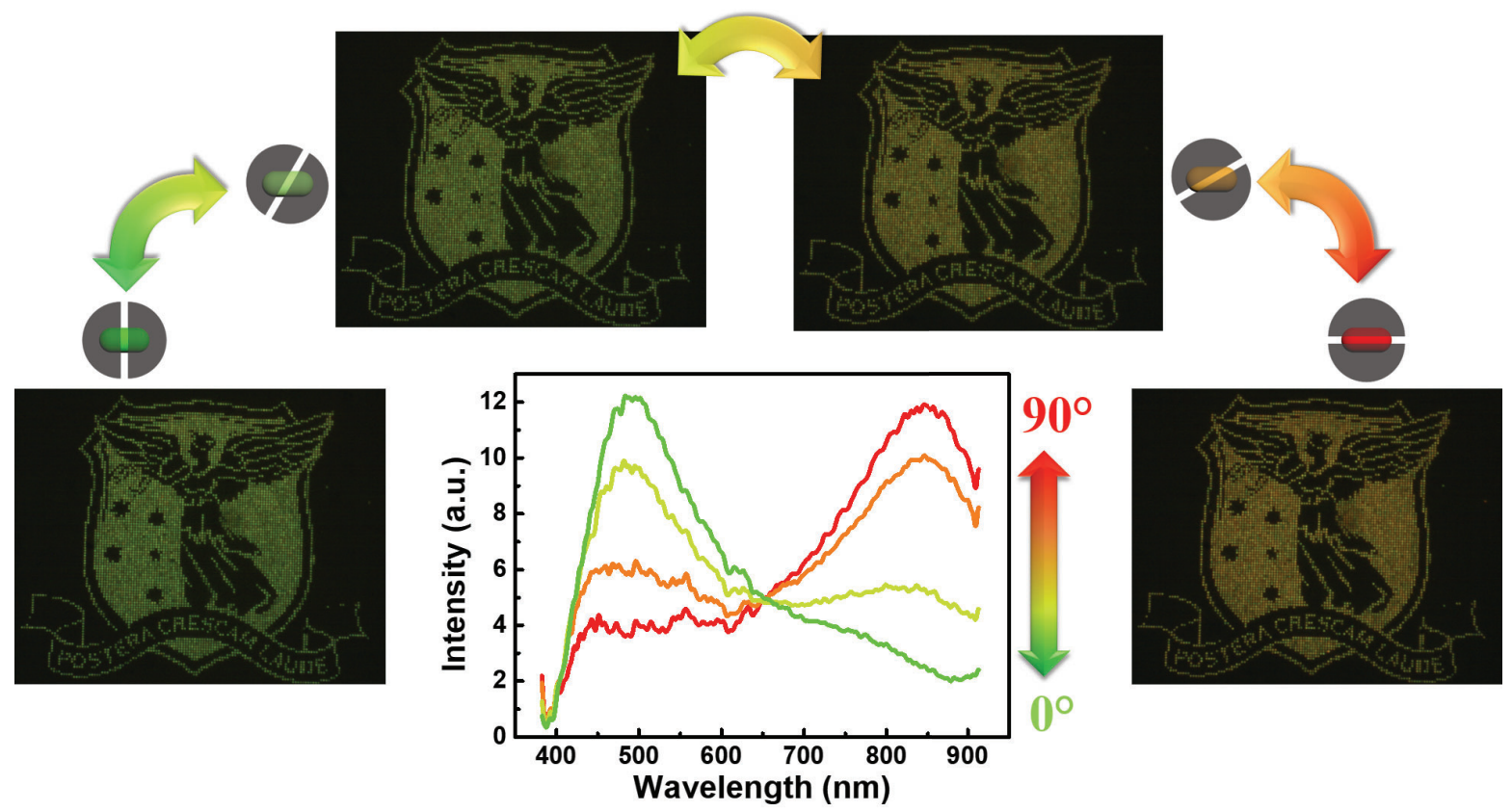

Figure S7. Dark-field scattering spectra of the logo of the University of Melbourne under 0 (Green), 30 (Yellowish green), 60 (Orange) and 90 (Red) polarization angle and corresponding dark-field images. 


\section{Large area "Au" logo}

a

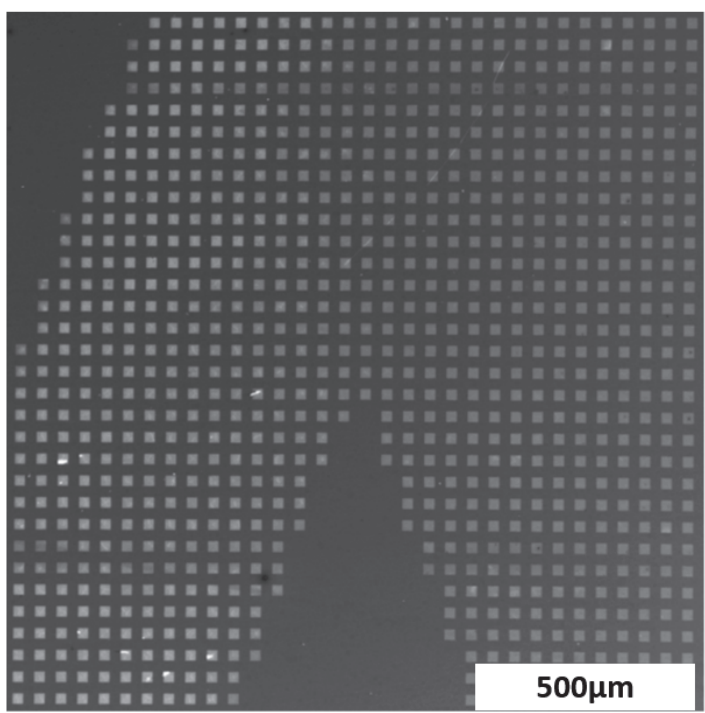

b

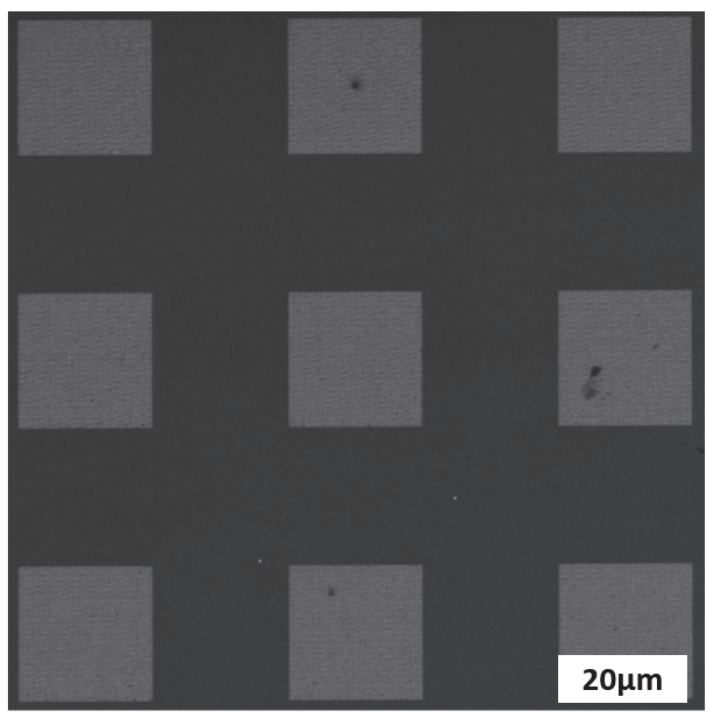

Figure S8 Scanning electron microscopy images of "Au" logo filled by gold NRs via EPD assembly. (a) Top part of the "Au" logo of the letter "A". (b) 9 of the Pixels in "Au" logo filled by gold NRs. 